\title{
Reicht Röntgen bei V. a. Hüft- und Beckenfrakturen?
}

\author{
Eine amerikanische Studie zeigt: \\ Auf Röntgenaufnahmen sind \\ Hüft- und Beckenfrakturen oft nicht \\ zu erkennen. Auch falsch-positive \\ Röntgenbefunde sind keine Selten- \\ heit.
}

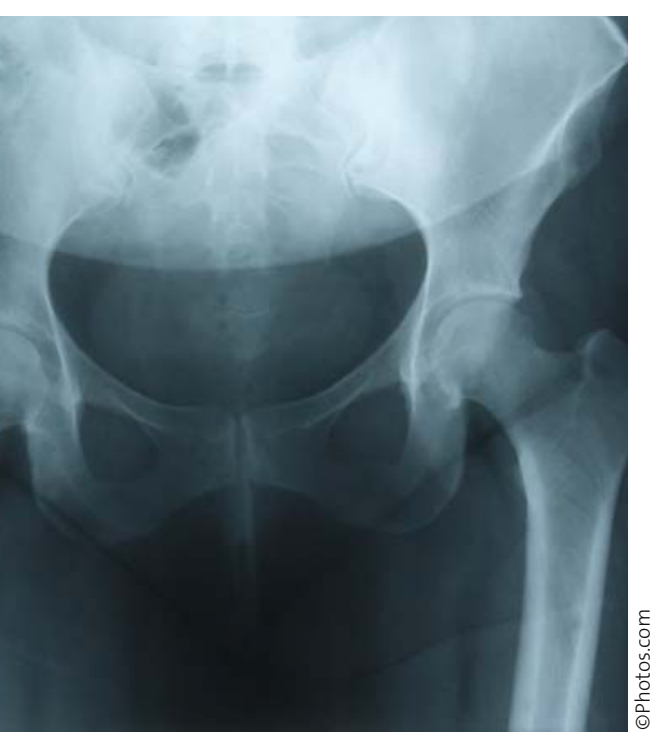

- 77 Frauen und 15 Männer (Durchschnittsalter 70,8 Jahre), die mit starken Schmerzen im Becken- und Rückenbereich die Notfallabteilung der Duke University in Durham aufsuchten, wurden routinemäßig auf Knochenbruch geröntgt. In einem zweiten Gang wurden alle mit der Magnetresonanztomografie (MRT) untersucht. Die Bilder wurden von zwei Radiologen ausgewertet, die Ergebnisse der beiden Diagnosetechniken verglichen.

Die MRT fand bei 35 Patienten, die laut Röntgen keinen Knochenbruch hatten, doch eine Fraktur. Umgekehrt hatten elf Patienten, bei denen der Röntgenbefund den Verdacht auf eine Fraktur nahelegte, eine negative MRT.

4 Beckenübersichtsaufnahme: Verbirgt sich hier eine Fraktur?

\section{Kommentar}

Die Hälfte der Röntgenbefunde war falsch negativ oder falsch positiv. Die Autoren schließen daraus, dass das Röntgen in der Notaufnahme zu ungenau ist, um die Ursachen von Hüft- und Beckenschmerzen zu beurteilen. Zum Ausschluss einer Knochenfraktur ist die MRT eindeutig sicherer und genauer als das Röntgen.

Ob sich diese Erkenntnis in der Praxis durchsetzen kann, ist allerdings fraglich. Die Röntgenuntersuchung ist wesentlich preisgünstiger. Andererseits sollte beachtet werden, dass bei der MRT die Strahlenbelastung wegfällt. Außerdem sind in der Regel kontrollierende Nachuntersuchungen bei unsicheren Befunden teurer als die teure, aber qualitativ bessere Primäruntersuchung.

K. MALBERG =

\section{- M. W. Kirby, C. Spritzer}

(Korrespondenz: C.Spritzer, Dept. of Radiology, Duke University Medical Center, Durham, NC 27710, USA) Radiographic detection of hip and pelvic fractures in the emergency department. Amer. J. Roentgenol. 194 (2010) 1054-106o

\section{Aggressiveres Prostata-Ca. bei unfruchtbaren Männern}

\begin{abstract}
Ob ein Zusammenhang zwischen der Unfruchtbarkeit beim Mann und dem Prostatakarzinomrisiko besteht, war bisher nicht ganz klar. Entsprechende Studien hatten widersprüchliche Ergebnisse geliefert.
\end{abstract}

- Nach Ansicht der Forscher von der University of Washington, Seattle (USA), war in den bisherigen Studien die Unfruchtbarkeit der Probanden nicht genau bestimmt worden. Als „unfruchtbar“ galten Männer, die keine Kinder gezeugt hatten. Für eine neue Studie wurden jetzt die Daten von 22562 Männern ausgewertet, die sich an 15 Zentren in Kalifornien auf ihre Zeugungsfähigkeit hin hatten untersuchen lassen.

168 dieser Männer hatten ein Prostatakarzinom. Damit war die Prostatakarzinomhäufigkeit unter den unfruchtbaren Männern nicht höher als unter einer vergleichbaren Population an fruchtbaren Männern. Qualitativ zeigte sich aber ein signifikanter Unterschied zwischen den beiden Gruppen: Hochaggressive Prostatakarzinome waren bei Männern mit nachgewiesener Unfruchtbarkeit 2,6-mal häufiger als bei der fruchtbaren Vergleichspopulation.

\section{Kommentar}

Zeugungsunfähige Männer haben ein fast dreimal größeres Risiko, an einem hochaggressiven Prostatakarzinom zu erkranken. Sie sollten deshalb früh in ein Screeningprogramm für Prostatakarzinome aufgenommen werden.

K. MALberG a

- T. J. Walsh et al.

(Urology Clinic, University of Washington Medical Center, 1959 N.E. Pacific St., Seattle, WA 98195, USA) Increased risk of high-grade prostate cancer among infertile men. Cancer, published online 22 March 2010; DOI: 10.1002/cncr.25075 